\title{
Formación en investigación educativa en la sociedad digital. Una experiencia innovadora de enseñanza en el nivel superior en el contexto latinoamericano
}

\section{Teaching Educational Research in Digital Society. An Innovative Teaching Experience at University Level in Latin American Context}

\author{
Selin Carrasco \\ Universidad Nacional de San Luis. Argentina. \\ selin.carrasco@gmail.com \\ Silvia Baldivieso \\ Universidad Nacional de San Luis. Argentina. \\ silvia.baldivieso@gmail.com \\ Lorena Di Lorenzo \\ Universidad Nacional de San Luis. Argentina. \\ lorenanataliadilorenzo@gmail.com
}

\begin{abstract}
Resumen
Desde la Cátedra Investigación Educativa II de la Universidad Nacional de San Luis, Argentina, nos preguntamos acerca de la pertinencia de los contenidos y valor de las estrategias de enseñanza que se utilizan para formar en investigación a los futuros profesionales de la educación y asumimos el reto de repensar la disciplina misma y su enseñanza en el contexto de la sociedad actual.

Se analizó la investigación a la luz de las nueva tecnologías y el desarrollo de lo digital, se indagó la expresión que esto adopta en el contexto latinoamericano y se planificó una experiencia de trabajo innovadora para los estudiantes de cuarto año del Profesorado y la Licenciatura en Ciencias de la Educación, con un enfoque de enseñanza centrado en el estudiante, y acciones que devienen de los principios del aprendizaje invertido, aprendizaje extendido y trabajo colaborativo.

El artículo profundiza la relación que se establece entre investigación educativa, tecnología y enseñanza de la investigación; y promueve la reflexión en torno al impacto que la misma puede tener sobre los contenidos de la formación de investigadores, los procesos de formación, el perfil de los investigadores y la investigación educativa misma. Relata los principios de los que se partió en la experiencia, el modo como se trabajó, los aspectos más valorados y los considerados críticos o que requieren más atención y estudio. Cierra con unas reflexiones generales.
\end{abstract}

\section{Palabras clave}

Investigación educativa, formación de investigadores, sociedad digital.

\footnotetext{
Abstract

From the Educational Research 2 Chair, at Universidad Nacional de San Luis, Argentina, we wonder about content relevance and value of teaching strategies used in the education of future educational professionals and we assume the challenge to rethink the discipline and its teaching methodology, in today's society context.

We analized research from new technologies aspects and digital development. The expression it takes in Latin American context was analyzed. We applied an innovative work
} 
experience to fourth year students of Educational Sciences, with a focus on studentcentered teaching and actions that arise from flipped learning, extended learning and collaborative work principles.

The article explores the relationship established between educational research, technology and educational research; and it promotes reflection on the impact it may have on the contents of training researchers, training processes, profile of researchers and educational research itself. The article describes the principles which originate the experience, the way we worked, the more valued and critical aspects considered or those which will require more attention and study. The article finishes with some general thoughts.

Key words

Educational research, researchers education, digital society.

\section{Introducción}

Desde la Cátedra Investigación Educativa II de la Universidad Nacional de San Luis, nos preguntamos acerca de la pertinencia de los contenidos y valor de las estrategias de enseñanza que se utilizan para formar en investigación a los estudiantes de pedagogía y asumimos el reto de repensar la disciplina misma y su enseñanza en el contexto de la sociedad actual y en América Latina.

Conscientes del valor que esta instancia de formación tiene para la renovación y recreación del espacio educativo, y de la necesidad de visualizarla más allá de las formulaciones teórico prácticas tradicionales; comenzamos a gestar otras miradas sobre el saber que nos ocupa, a buscar nuevas estrategias para transformar la dinámica tradicional de construcción de conocimiento en el campo educativo e investigativo y a iniciar a los estudiantes en prácticas renovadas y concepciones creativas de lo metodológico. Siempre a la luz de las potencialidades del entorno digital y las transformaciones del objeto de investigación en el campo educativo.

Nuestra preocupación deviene de visualizar que la sociedad contemporánea es una sociedad compleja y en transformación y el cambio que vivimos, genera una nueva forma de expresión que se manifiesta en todos los ámbitos, y con especial fuerza en el educativo.

Nuestra apuesta es considerar lo antes dicho para replantear la investigación educativa y la forma de enseñarla.

El presente trabajo realiza una revisión de la relación investigación educativa, tecnologías de la información y la comunicación (TIC) y enseñanza de la investigación en instituciones latinoamericanas; una reflexión general sobre la tradición de enseñanza de la investigación, y la necesidad de desarrollar un nuevo corpus de conocimientos teórico-didácticos, para formar investigadores y analiza una experiencia innovadora en el espacio de la formación de grado.

Para dar cuenta de lo trabajado durante el año 2015 se sitúa la experiencia en su contexto latinoamericano e institucional, se especifican los principios y fundamentos que la orientaron, se detallan aspectos vinculados a las actividades desarrolladas y se realizan algunos análisis de resultados y desafíos venideros en esta línea de trabajo, para cerrar con una reflexión general.

Formación en investigación educativa en la sociedad digital. Una experiencia innovadora de enseñanza en el nivel superior. Selín Carrasco, Silvia Baldivieso y Lorena Di Lorenzo. 
Concebimos el aprendizaje de la investigación como una instancia de problematización en torno a la realidad educativa; de concientización sobre la estrecha relación que establece con concepciones epistemológicas y como una instancia de formación en metodología de la investigación, es decir en el conjunto de principios, procesos y procedimientos que posibilitan relacionar críticamente epistemología, método, técnicas de investigación y tecnologías, con vistas a la construcción de conocimiento

\section{Investigación educativa, tecnologías y enseñanza de la investigación en instituciones latinoamericanas.}

La investigación educativa representa un conjunto de prácticas sociales que tienen lugar en contextos socio históricos y marcos políticos e institucionales que la condicionan y le dibujan perfiles específicos.

Se caracteriza por ser un campo en tensión, atravesado por criterios y procedimientos del ámbito de la investigación científica, como así también por demandas de la política pública y demás actores del sistema educativo (Tedesco, 2006). Como expresa Palamidesi et al. (2014) refiriendo a Diaz (1995), se trata de múltiples espacios de producción de discurso que no corresponden al modelo típico de las disciplinas científicas o de comunidades científicas claramente reconocibles, delimitadas y formalizadas, sino a áreas con contornos imprecisos y conexiones cambiantes, que funcionan en distintos niveles y jerarquías; lo que para Becher (2001) significa que está constitutivamente atravesada por racionalidades, intereses y peticiones de legitimidad diversas.

Presenta también la complejidad propia de la investigación social, que desafía a trabajar en la aparente contradicción de la focalización de la complejidad, por su estrecha relación con el contexto que da cuenta de los factores que la condicionan y sirven de anclaje a las decisiones del investigador. (Sirvent, 2015)

Analizada en si misma, la investigación educativa asume la complejidad propia de su objeto de estudio, la que le confieren las diversas perspectivas epistemológicas, y la que se genera en el inevitable enlace estructural con los ámbitos donde se define, sucede y administra la educación.

Analizada en la perspectiva regional o latinoamericana la investigación educativa expresa la complejidad de una territorio heterogéneo que a pesar de contar con no pocas características comunes posee importantes diferencias étnicas, inmigratorias, de historia política, económica cultural, etc. que en el decir de Narodowsky (1999), dejan dudas respecto a la posibilidad de hablar de una "identidad" en la investigación educativa.

"Si bien un pequeño grupo de países ha logrado una incipiente institucionalización (...) el resto de los países todavía carece de lo fundamental para construir esta área como campo de estudio, debido a una limitada producción de investigaciones así como la inexistencia de centros de investigación y/o formación específica para desarrollarse autónomamente en el área del conocimiento." según expresa Narodowski (1999) citando a Garcìa-Guadilla (1998), que refiere a las investigaciones sobre educación superior.

Asimismo, la región presenta en la actualidad un panorama caracterizado por un proceso de crecimiento económico y de lento pero consistente avance de los regímenes

Formación en investigación educativa en la sociedad digital. Una experiencia innovadora de enseñanza en el nivel superior. Selín Carrasco, Silvia Baldivieso y Lorena Di Lorenzo. 
democráticos y de los procesos de modernización social e institucional. En ese contexto, los sectores de la educación y de ciencia y tecnología han experimentado un aumento significativo de la inversión pública. A su vez, se ha impulsado el desarrollo de sistemas nacionales de investigadores y la vinculación con redes internacionales de intercambio científico-técnico (Didou-Aupetit, 2007)

Estudios recientes realizados sobre investigación educativa ponen en evidencia interesantes desarrollos y producciones con ritmos propios en los diferentes países.

Palamidessi, et al. (2014) analiza como la producción de conocimientos en educación en América Latina se asocia - en forma bastante directa - con los niveles de diferenciación e institucionalización de los sistemas de educación superior y de las políticas y agencias estatales de promoción de ciencia y tecnología en cada país. Estos distintos grados de desarrollo, a su vez, están asociados al mayor o menor grado de profesionalización del campo académico en educación y ciencias sociales y de las tecno-burocracias de los sistemas educativos y considera los casos de Brasil, México, Chile, Argentina, Uruguay y Paraguay, reconociendo el desarrollo de los dos primeros, la tendencia a la estructuración de Chile, los desarrollos de Argentina y Uruguay (salvando las diferencias de tamaño) y la escasa articulación de Paraguay. Destacan que estos últimos países probablemente ilustran los rasgos predominante en el resto de la región.

En el espacio centroamericano destacan los desarrollos y producciones de Costa Rica. Solís, M et al. (2012) dan cuenta de esto en un trabajo que analiza la investigación educativa en los procesos de formación inicial docente de primaria en universidades públicas y privadas y destaca la creciente importancia que esta, está adquiriendo en el contexto nacional y regional.

A los estudios y mediciones que posicionan los aportes, le sucede la necesaria reflexión respecto a la validez de los indicadores y su significatividad en relación al alcance y aportes que las producciones realizan, su focalización en las problemáticas reales y su compromiso con la realidad que demanda.

En el campo de la educación y la investigación, América Latina de la mano de Paulo Freire y los desarrollos en educación popular y educación de adultos, ha realizado interesantes aportes en la línea de investigación acción participativa y de sistematización de experiencias como forma de investigación social.

\section{En lo que respecta investigación y tecnología}

No se dispone de estudios sistemáticos que den cuenta del estado de imbricación de la tecnología en el desarrollo de la investigación educativa en la región, pero sí de evidencias que muestran la articulación de los países que buscan fortalecerse y promover desarrollos en y desde la ciencia y la tecnología. Como por ejemplo el caso de la Red Clara (www.redclara.net) cuya misión es fortalecer el desarrollo de la ciencia, la educación, la cultura y la innovación en América Latina a través del uso innovador de redes avanzadas" y se proyecta al 2017 como un actor clave en el fortalecimiento de la ciencia y la tecnología en América Latina

Formación en investigación educativa en la sociedad digital. Una experiencia innovadora de enseñanza en el nivel superior. Selín Carrasco, Silvia Baldivieso y Lorena Di Lorenzo. 
La Referencia, iniciativa regional conformada en 2012 por nueve países socios que participan activamente, representados por Organizaciones Nacionales de Ciencia y Tecnología de: Argentina, Brasil, Chile, Colombia, Ecuador, El Salvador, México, Perú y Venezuela; a la que se acaba de incorporar Costa Rica como socio observador.

La misma busca compartir y dar visibilidad a la producción científica generada en las instituciones de educación superior y de investigación científica en América Latina, gracias a la creación de un marco de acuerdos técnicos y organizativos para construir la Red Federada de Repositorios Institucionales. (lareferencia.net) y a la fecha ha relevado mas de cien repositorios de catorce países de América Latina, en su mayoría registrados en los Directorios Internacionales.

El caso de RNIE cuyo objetivo es el fortalecimiento de las redes Nacionales de Investigación y Educación de Mesoamérica, y por nombrar algunas de las redes de tecnología y educación para el desarrollo más reconocidas, RENATA de Colombia, REUNA de Chile, INOVARED de Argentina, RNP de Brasil, CUDI de México , REDICCES de El Salvador, CONARE de Costa Rica, etc.

Cabe destacar en este apartado la preocupación por la incidencia de la investigación en el espacio educativo y la necesidad buscar nuevas herramientas para comprender y contribuir a resolver los problemas de un mundo que está cambiando velozmente, de relevar los dilemas y necesidades de este momento, de promover la utilización de la investigación existente, de incluir nuevos usuarios del conocimiento producido en los espacios académicos mediante dispositivos específicos que favorezcan dicho acercamiento utilizando los soportes disponibles (Tedesco, 2006).

Cabe también resaltar que la incorporación de tecnologías por parte de los investigadores en diferentes momentos del proceso de investigación es una realidad en crecimiento. Evidencia de ello se encuentra en el trabajo de Hernández et al. (2013) que indagan el uso de las tecnologías entre los investigadores educativos en México destaca el acceso a las TIC al momento de recabar información, en la comunicación por correo electrónico y en el análisis cuantitativo de datos fundamentalmente.

\section{En lo que respecta a la formación de investigadores}

Nos interesa destacar junto a Rojas-Soriano (2015) que la investigación es un proceso dialéctico ya que a través de ella busca reconstruirse en el pensamiento, una realidad objetiva que se desenvuelve dialécticamente, no de manera lineal, mecánica. Por lo mismo, no hay esquemas o modelos de investigación únicos y definitivos sino solo guías que orientan el desarrollo del trabajo de investigación, las cuales se ajustan a los requerimientos que exige la práctica científica en cada situación concreta. Esta concepción de la investigación permite superar la posición reduccionista que todavía prevalece en muchas instituciones de educación superior en donde se piensa que contar con un esquema o una receta es suficiente para alcanzar la verdad científica.

Asimismo existe una visión compartida respecto a la necesidad de repensar la formación en la región y algunas reflexiones y propuesta que señalan rumbos.

El mismo autor anteriormente citado realiza algunas reflexiones teóricas entre las que destacan la necesidad de mantener contacto con la realidad; de mantener a la práctica

Formación en investigación educativa en la sociedad digital. Una experiencia innovadora de enseñanza en el nivel superior. Selín Carrasco, Silvia Baldivieso y Lorena Di Lorenzo. 
como criterio de verdad para mostrar la validez de los planteamientos, de construir conocimiento y transformar la realidad; de relevar el carácter socio histórico y objetivosubjetivo de la investigación social, de considerar la lógica interna y consecuentes leyes que rigen el proceso de investigación; la exigencia de vinculación directa y permanente de los elementos filosóficos, teóricos, metodológicos y técnico-instrumentales; el necesario apoyo que el proceso de formación debe tener en una ciencia critica de la educación; la necesidad de trabajo interdisciplinario; de participación en talleres; la preparación para exponer los resultados de la investigación; etc.

Rincón (2004) en un estudio sobre la situación de la formación, los retos y perspectivas para América Latina en el siglo XXI, señala la necesidad de formar investigadores en América Latina como estrategia para afrontar los problemas que subyacen a la educación. Desde su perspectiva, la formación de investigadores en educación debe diseñarse como una propuesta alternativa de profesionales que, además de atender con profesionalismo los problemas educativos, adquieran un compromiso social y humano que les permita participar en la formación de los más altos valores universales.

Analiza como la formación de investigadores en educación en América Latina, se encuentra precedida por el desarrollo de los campos disciplinarios que abordan a la educación como su objeto de estudio y por la presencia de modelos educativos neoliberales. Por lo tanto, también el estado actual de la investigación educativa en los países, se encuentra influido por las formas en que las diferentes disciplinas científicas, incluidas las llamadas ciencias de la educación, han logrado un nivel de consolidación teórico y metodológico en la búsqueda de encontrar soluciones a los problemas educativos.

Destaca como la producción del conocimiento educativo, tiene que enfrentar los desafíos de los grandes cambios en la ciencia, la técnica y la sociedad. Cambios que exigen reorientar los procesos de formación de investigadores. Y requieren la integración inter y multidisciplinaria de los diferentes campos científicos que han abordado a lo educativo como objeto de estudio, y la colaboración colegiada de equipos de académicos provenientes de diferentes campos, para formar a los investigadores de la educación desde el interior de los problemas mismos y no desde posiciones que se encuentren fuera de los mismos.

Sánchez-Puente (2014), propone una nueva didáctica de la investigación científica que promueve el cambio en el marco de una planeación estratégica adecuada a los destinatarios.

Reconoce tres maneras de enseñar a investigar, la centrada en la ciencia, (de corte epistemológico) a la cual cuestiona destacando que el concepto de ciencia es un concepto histórico, porque designa diferentes prácticas de la ciencia a lo largo de la historia ; la centrada en el estudio de la estructura de la investigación que trata de enseñar a identificar los elementos constitutivos y las relaciones de la estructura; y la que recupera la dimensión dinámica del quehacer científico asumiéndose la investigación como proceso y orientando la enseñanza a un conjunto de pasos que hay que atravesar hasta llegar al conocimiento buscado. La nueva didáctica propone asumir los quehaceres y operaciones mismos de la investigación y generación de conocimiento como contenidos del proceso. 
Sirvent (2015) analizando la formación de investigadores en el post grado señala que tanto en las instituciones científicas como en las instituciones de educación superior (de América Latina) se observa con preocupación la presencia cotidiana de mecanismos de poder y de toma de decisiones institucionales que obstaculizan la generación de ámbitos de debates y discusión plenamente democráticos y que por tanto "hieren" la formación de una ciudadanía democrática. Considera que varios de los parámetros y criterios de evaluación de las políticas científicas son "anticientíficos" porque no facilitan la generación de las condiciones objetivas necesarias para la formación en el oficio de investigador y para el crecimiento de los investigadores jóvenes en el alma y el corazón de la ciencia: la creatividad, la libertad, la autonomía y el pensamiento reflexivo y crítico, señalando la preocupación por el impacto negativo de estas políticas científicas en la formación de las nuevas generaciones de jóvenes científicos.

Afirma que en términos generales en América Latina estamos enfrentando un contexto de lucha social y académica por una sociedad más justa e igualitaria y una ciencia emancipadora y propone tres desafíos de índole metodológico, para una metodología de la investigación social y didáctico, para una práctica pedagógica.

- la génesis de una investigación en cuanto el contexto socio-histórico y su función.

- la génesis de una investigación en cuanto a la problematización y su función

- la complejidad de la naturaleza de la investigación de lo social

Como se puede observar en este marco de las preocupaciones por la formación en investigación en América Latina, las tecnologías de la información y la comunicación y la transformación que ellas provocan en los procesos de enseñanza de la investigación y en los contenidos disciplinares mismos no cuentan con un lugar específico, y muchas veces ni siquiera tienen lugar; y si participan lo hacen desde el espacio que les concede la práctica pedagógica, no desde la búsqueda de una nueva metodología de la investigación.

Salvo contadas excepciones en el contexto, las tecnologías se consideran sólo herramientas desconociendo que las mismas son también el entorno vital constitutivo de los sujetos y procesos y la relación dialéctica que se establece con el mismo en el espacio educativo. $\mathrm{Si}$, se han desarrollado algunas experiencias aisladas que intentan articular los tres elementos en análisis (investigación educativa, formación de investigadores y tecnologías de la información y la comunicación), como el trabajo que presenta Piñero L. et al. (2010) que en un proceso de trasformación y modernización del currículo para la formación docente de pregrado de la Universidad Pedagógica Experimental Libertador, destaca la consideración de las TIC y la investigación como ejes transversales como marco de una metodología integradora dentro del proceso de formación del futuro docente.

\subsection{Tradición de formación en investigación en la universidad argentina}

En este apartado destacamos que en la formación tradicional en investigación en las universidades argentinas se ha caracterizado por una fuerte tradición positivista, que durante décadas se concentró en la enseñanza de la metodología cuantitativa con énfasis en las estadísticas.

Formación en investigación educativa en la sociedad digital. Una experiencia innovadora de enseñanza en el nivel superior. Selín Carrasco, Silvia Baldivieso y Lorena Di Lorenzo. 
Esta tradición en la formación investigativa (visualizable en requisitos de tesis, becas de investigación y materias metodológicas) se edificó a partir del modelo canónico y la consiguiente preeminencia del positivismo. Actualmente se siguen priorizando los enfoques cuantitativos y las pocas investigaciones cualitativas son generalmente verificacionistas ${ }^{1}$, reproduciendo las teorías que los estudiantes han visto durante su formación de grado.

Este modelo hegemónico para formular una investigación, en términos de Boaventurade-Sousa (2009) el paradigma dominante de hacer ciencia, brinda sus requisitos de objetividad, rigurosidad y protocolo claramente establecidos, los cuales hay que seguir al pie de la letra para obtener el grado o posgrado académico, en consecuencia la enseñanza de la metodología se limita a transmitir dichas reglas.

Las universidades en el contexto neoliberal han contribuido a la consolidación de un modelo instrumental y apolítico en las formas de llevar a cabo investigaciones científicas y de enseñar la metodología. En este sentido afirma Sirvent (2015:1) "varios de los parámetros y criterios de evaluación de nuestras políticas científicas son "anticientíficos" porque no facilitan la generación de las condiciones objetivas necesarias para la formación en el oficio de investigador y para el crecimiento de nuestros investigadores jóvenes en el alma y el corazón de la ciencia: la creatividad, la libertad, la autonomía y el pensamiento reflexivo y crítico".

El mencionado modelo instrumental se traduce en una enseñanza vertical y lineal de las reglas, instrumentos y técnicas a aplicar para llevar adelante una investigación.

La formación Tradicional propicia una fuerte separación entre teoría y práctica. Por un lado las clases teóricas en las cuales se transmiten las técnicas, los procedimientos para diseñar y poner en marcha una investigación y por otro lado la práctica donde se espera que el alumno "lleve a la práctica" dichas reglas, dividiendo la formación en investigación en dos partes no retroalimentadas, sino que una es condición para la otra.

La reflexividad, por el contrario, ubica la metodología en un lugar secundario, ya que esta no determina el tipo de trabajo, solo facilita, dificulta o posibilita la reflexividad como forma de construcción de conocimientos con otros, a partir de una comunicación no violenta y sin directrices, ubica a la subjetividad como requisito de objetividad.

\section{Transformación del contexto de la investigación y su impacto sobre las posibilidades de la investigación y su enseñanza.}

En este apartado focalizamos la investigación en el campo de la educación a la luz de las transformaciones del contexto en la que la misma tiene lugar y relevamos las potencialidades y desafíos que abre al espacio de la formación en investigación.

La investigación educativa en tanto practica social, que se genera cuando el investigador percibe una situación problemática y se interroga respecto a ésta; se desarrolla en estrecha relación con el contexto socio histórico en el que tiene lugar (Sirvent, 2006).

\footnotetext{
$1 \quad$ - Verificacionistas - refiere al modo verificativo de hacer ciencia de lo social, en contraposición a los modos de generación conceptual y participativo.
}

Formación en investigación educativa en la sociedad digital. Una experiencia innovadora de enseñanza en el nivel superior. Selín Carrasco, Silvia Baldivieso y Lorena Di Lorenzo. 
Es en un tiempo y en un espacio social determinado, con el conjunto de factores sociales, políticos, económicos, tecnológicos, institucionales, etc. que lo componen, (contexto de descubrimiento), donde surge un problema de investigación, y es en él también donde se desarrollan las condiciones que facilitan o limitan su abordaje.

Esta concepción de investigación cobra especial significación en el contexto de la sociedad actual, caracterizada por la impronta de los procesos de cambio y la nueva cultura que trae aparejada (Castells, 2008).

El desarrollo de la tecnología y la emergencia de un tercer entorno o sociedad digital (Echeverría, 2001), la complejidad e interrelación continua de sus partes (Morín, 1999); el triple rol que asumen las tecnologías en la sociedad contemporánea (Carrasco, 2011), la transformación en la formas de producir y difundir el conocimiento, el auge de los sistemas de formación en línea, de los contenidos abiertos, de los portales educativos, del e, b, m learning, de los MOOC, etc. transforman las posibilidades de las instituciones educativas, al tiempo que cuestionan las prácticas tradicionales.

La investigación educativa se ve impelida a repensarse como disciplina y tiene la oportunidad de hacerlo. Requiere de un nuevo marco conceptual epistemológico que valide las nuevas formas posibles de generar conocimiento en el entorno digital colaborativo; un nuevo marco teórico metodológico que guie la lógica procedimental conforme las nuevas posibilidades de construcción de conocimiento, requiere también capacidades y destrezas tecnológicas y procedimentales, etc. y por supuesto requiere un nuevo espacio y forma de trabajo para enseñar/aprender a investigar.

\section{Sobre la experiencia}

El trabajo se inscribe en la línea de sistematización de experiencias, concebidas estas como un proceso de investigación socio educativa, orientada a construir conocimiento a partir de la recuperación, la comprensión, la explicación de la práctica o de la experiencia vivida en un quehacer social, cultural o educativo. La sistematización se entiende como un "Proceso que parte de la práctica, reflexiona la práctica y produce saber para transformarla". (Messina, 2004) y que considera la experiencia como procesos vitales (socio históricos) dinámicos y complejos, individuales y colectivos vividos por personas concretas.

Conforme a ello y de acuerdo a las fases de esta modalidad investigativa, propia del campo social y educativo, se elaboró un perfil de sistematización, junto con la planificación de la asignatura, lo cual facilitó el proceso de recolección de información y reconstrucción, (cabe recordar que en esta modalidad investigativa, la sistematización puede realizarse al término de una experiencia o durante su desarrollo), se reconstruyó lo vivido, se contrastaron perspectivas e informaciones provenientes de diferentes fuentes (observaciones, análisis de documentos, entrevistas a miembros de la cátedra y a los estudiantes, etc.) y se dan a conocer los resultados. Resta aun trabajar en el rediseño de la experiencia a la luz de las reflexiones y conclusiones (aprendizajes alcanzados).

Formación en investigación educativa en la sociedad digital. Una experiencia innovadora de enseñanza en el nivel superior. Selín Carrasco, Silvia Baldivieso y Lorena Di Lorenzo. 


\subsection{Contexto institucional de la experiencia:}

La experiencia de formación se llevó a cabo en la asignatura Investigación educativa II del Profesorado y Licenciatura en Ciencias de la Educación en la Universidad Nacional de San Luis. La misma tiene lugar en el cuarto año de estudio y se desarrolla a lo largo de un año académico en cien horas presenciales de trabajo.

Los estudiantes, jóvenes entre 22 y 30 años aproximadamente acceden habiendo cursado y aprobado un par de asignaturas previas vinculadas a investigación y una referida a educación y medios, pero sin experiencia real de trabajo en estos temas.

La asignatura pertenece a un Plan de Estudios que se inicia en la universidad en 1999, con el espíritu de renovar la formación de pedagogos, rompiendo con la estructura y práctica del plan que le antecedió, (17/78) concebido durante la época de la dictadura militar y criticado por sus posturas tecnocráticas, cientificistas, y de corte positivista; con gran profusión de contenidos, material y cursos, que no articulaban la formación general con la especifica ni con la realidad (Di Lorenzo, 2015).

Bregando contra el peso de la tradición la propuesta integral de formación de pedagogos donde se sitúa investigación educativa II, generó posibilidades para flexibilizar las cátedras, integrar investigación, docencia y extensión; intensificar la relación teoríapráctica, y desarrollar trabajo interdisciplinario; buscando un profesional preparado para dar respuestas alternativas a las necesidades reales.

Aunque han trascurrido muchos años de aquella formulación inicial y el espíritu del plan se renueva continuamente al interior de la carrera, continua existiendo cierta dificultad para situar a los estudiantes en el contexto real de su formación y de prepararlos para que trabajen conforme las previsiones de desarrollo futuro. No siempre la formación está actualizada ni los estudiantes preparados para anticiparse. Aunque uno de los objetivos principales del currículum es formar para comprender la realidad educativa en su naturaleza dialéctica, compleja y situada históricamente para explicarla y transformarla.

La formación en metodología, en este espacio se organiza a lo largo de un eje o área de formación epistemológica metodológica que contempla las signaturas: epistemología de las ciencias sociales, investigación educativa I, investigación educativa II, praxis IV: la práctica investigativa y el taller de tesis.

Puede observarse que la formación en investigación educativa es muy fuerte en la carrera, al contar con 5 espacios curriculares abocadas a la misma. Sin embargo se visualizan actualmente profundas demoras en las realizaciones de las tesis de grado y dificultades teórico-prácticas en el campo de la investigación, lo que nos desafía a producir modificaciones en las formas de enseñar a investigar.

\subsection{Principios y fundamentos de la experiencia}

Desarrollamos la propuesta a partir de una premisa inicial "el desarrollo de las Tecnologías de la información y la comunicación (considerando su triple rol) y la consecuente sociedad digital en la que nos encontramos inmersos, transforman la

Formación en investigación educativa en la sociedad digital. Una experiencia innovadora de enseñanza en el nivel superior. Selín Carrasco, Silvia Baldivieso y Lorena Di Lorenzo. 
educación y transforman la investigación educativa exigiendo replantear la investigación y la forma de enseñarla".

La interrelación de las nuevas tecnologías influyen en la creación de una nueva sociedad, distinta de la industrial, conforman un nuevo espacio social el espacio electrónico o espacio digital llamado por Echeverría (2001) Tercer entorno. Este surge como un necesidad y se desarrolla de manera superpuesta sin que incida sobre los dos anteriores (campo y ciudad) pero si posibilitando múltiples y diversas formas de acción, como en el caso de la investigación científica que se ha adaptado fácilmente a este nuevo espacio. Los científicos hoy en día se comunican entre sí, intercambian preguntas, hipótesis, analizan, hacen experimentos a través de redes, etc.

Son las tecnología las que generan el nuevo espacio social, las que lo soportan y las que nos permiten acceder al mismo, sin embargo son mucho más que una herramienta que conecta.

En el ámbito de lo educativo, es fundamental trascender esta perspectiva instrumentalista y sostener una propuesta que considere la emergencia de la dimensión virtual y sus herramientas, que considere el triple rol que adquieren las TIC en la formación docente: a) contexto capaz de modificar los roles de los actores participantes en el proceso educativo y a los procesos mismos, b) objeto de estudio que se incorpora al currículo de la formación docente, y c) y herramienta que puede integrarse intencionada y reflexivamente a los procesos de enseñanza en pos de más y mejores aprendizajes y no como meras herramientas continuadoras de lo existente en la sociedad industrial, y educación tradicional (Carrasco et al, 2014).

Múltiples y diversas expresiones de nuevos procesos posibles ponen en evidencia la transformación educativa que las TIC traen consigo, por nombrar las más cercanas decimos, enfoque flexible, centrado en el estudiante, aprendizaje autorregulado, ubicuo, educación en línea. entornos personales de aprendizaje, clase invertida, etc. Cada una de ellas desplegándose en el mismo entorno pero poniendo en juego prácticas y capacidades diferentes.

Todos los cambios antes mencionados, especialmente los que tienen lugar en el ámbito de lo educativo, nos significan transformaciones en la investigación educativa como práctica (investigativa) y como contenido (de la formación en investigación). y replanteamientos en los procedimientos de investigación y en la forma de enseñarla. En tal sentido destacamos:

Transformaciones de la investigación educativa

- En el objeto de estudio de la investigación educativa: es decir, cambios en la educación y en los procesos de enseñanza aprendizaje que exigen diversidad de estrategias, medios, para acceder a ellos.

- En la práctica investigativa, es decir, desarrollo de nuevos procesos, procedimientos e instrumentos para indagar las aristas que el objeto cobra en el nuevo entorno ( $o$ del nuevo objeto)

- En la oportunidad de desarrollar nuevos procesos y procedimientos, de investigación asociados al desarrollo tecnológico; es decir procesos y procedimientos compatibles con el nuevo entorno y con la expresión del objeto de investigación.

Formación en investigación educativa en la sociedad digital. Una experiencia innovadora de enseñanza en el nivel superior. Selín Carrasco, Silvia Baldivieso y Lorena Di Lorenzo. 
- En la emergencia de nuevos conceptos que explican y fundamentan la investigación educativa.

Replanteamiento de los Contenidos y Procesos de la Investigación Educativa

- Para constituir equipos de investigación (redes de investigadores).

- Para construir objetos (particularidades del contexto de descubrimiento).

- Para formular marcos teóricos, y Estados del Arte: (gestores de información científica, acceso abierto, etc.).

- Para recoger información (instrumentos digitales, sistemas de registro, etc.).

- Para analizar la información (softwares especializados).

- Para discutir los resultados (redes y softwares especializados).

- Para comunicar la ciencia (espacios, medios y recursos disponibles).

- Para garantizar la calidad de la investigación (redes, espacios, medios y recursos)

- Etc.

Replanteamiento de la forma de enseñar la investigación educativa

Nos replanteamos la enseñanza de la investigación educativa, a partir de la propuesta curricular, orientándonos hacia la búsqueda de un egresado atento a la demandas actuales propias de una sociedad dinámica, diversa, contradictoria, conflictiva, y comprometiéndonos con la formación de sujetos problematizadores de la complejidad educativa, capaces de abordarla desde una óptica flexible, creativa y comprometida; profesionales preparados para leer y actuar en el escenario actual, haciendo uso de todo el potencial de los elementos que este ofrece, dando respuestas y anticipándose.

\subsection{Objetivos de la experiencia}

A fin de dar continuidad y consistencia al eje de análisis sostenido, en este trabajo presentamos solo aquellos objetivos vinculados al tema que nos ocupa (la investigación en el contexto de lo digital)

En presentaciones posteriores daremos cuenta de otros elementos asociados a una trasformación integral en la forma de enseñar la metodología de la investigación (como la dimensión teórico epistemológica de los contenidos y su anclaje en el contexto latinoamericano, la dimensión política institucional y los asociados a la didáctica de la investigación propiamente dicha).

En esta ocasión relevamos aquellos que sitúan la investigación en el contexto de lo digital.

- Objetivo 1: Visualizar la metodología de la investigación educativa en el marco de las problemática de la práctica docente y las transformaciones socio educativas de la sociedad actual.

- Objetivo 2: Analizar críticamente el impacto de las tecnologías de la información y la comunicación sobre los procesos de construcción de conocimiento en educación.

- Objetivo 3: Propiciar el desarrollo de nuevas estrategias y técnicas de investigación para el desarrollo de la investigación educativa en el mundo digital

Formación en investigación educativa en la sociedad digital. Una experiencia innovadora de enseñanza en el nivel superior. Selín Carrasco, Silvia Baldivieso y Lorena Di Lorenzo. 


\subsection{Propuesta pedagógico tecnológica}

Para aproximarnos a los objetivos propuestos nuestra propuesta pedagógicotecnológica adoptó elementos del extended learning, blended learning, flipped learning y trabajo colaborativo.

Se basó en el desarrollo de un curso de modalidad presencial extendida mediante las TIC. Extendimos la clase y las posibilidades de trabajo más allá de los límites temporales y espaciales determinados por el plan y la tradición institucional, tal como lo promueve (González, 2012). Esta extensión se realizó a partir de la incorporación de un Entorno Virtual de Aprendizaje (aula virtual de la UNSL) que opero como una puerta de entrada al mundo digital.

Las estrategias de trabajo en este aula extendida se diseñaron con la lógica de la clase invertida en la que la instrucción directa se mueve desde el espacio de aprendizaje colectivo hacia el espacio de aprendizaje individual, y el espacio resultante se transforma en un ambiente de aprendizaje dinámico e interactivo en el que el educador guía a los estudiantes a medida que se aplican los conceptos y puede participar creativamente en la materia (Aaron, 2014); y con la lógica del trabajo colaborativo y la producción conjunta que propusieron trabajos que procuraban el desarrollo de capacidades individuales y grupales.

Todo orientado por la búsqueda de flexibilidad en el proceso promoviendo la autonomía para decidir su aprendizaje, (aunque sin regirnos por los principios del aprendizaje abierto o flexible); y desarrollado bajo un enfoque de enseñanza centrado en el estudiante, es decir, con reemplazo de la tradicional práctica de "dar" contenidos por estrategias de enseñanza orientadas a ayudar a aprender, a promover actividades que involucraran a los estudiantes en sus aprendizajes. (Baldivieso, 2012)

De este modo se fue comprometiendo a los estudiantes con su proceso de aprendizaje; se abrieron espacios de trabajo adaptables donde los alumnos eligieron cuándo y a través de qué aprender (con un requisito mínimo de cumplimiento al término del año); se promovieron trabajos individuales y grupales, con consignas que contribuyeron a que la construcción grupal fortaleciera las capacidades individuales y viceversa, etc.

En aspectos generales se fueron propiciando otras formas de vínculo con el contenido, con los docentes y entre los estudiantes: se fueron desarrollando actividades de enseñanza y de aprendizajes diferentes (acordes al cambio de la educación) y actividades de investigación diferentes: acordes al desarrollo de la investigación en el entorno actual.

Algunas actividades interesantes desde el punto de vista del aprendizaje, fueron las siguientes:

- Gestión en línea de información científica descubriendo y aprendiendo previamente el uso de motores de búsqueda, gestores bibliográficos, etc. (en instancias de formación y desarrollo de habilidades tecnológicas).

- Producción de imágenes/representaciones de conocimiento/videos: (en instancias de integración).

- Glosario y repositorio construido colectivamente: para fortalecer los conocimientos y construir marcos teóricos de la asignatura.

Formación en investigación educativa en la sociedad digital. Una experiencia innovadora de enseñanza en el nivel superior. Selín Carrasco, Silvia Baldivieso y Lorena Di Lorenzo. 
- Foros por temas: para apropiarse de los contenidos y construir conocimientos colectivamente.

- Foros transversales anuales para integrar y complejizar los análisis a partir del desarrollo gradual e interrelación constante de los temas.

- Mapas conceptuales para reconstruir la materia interrelacionando cada uno de los temas.

- Visualización de nuevos instrumentos de recolección de información posibles de desarrollarse/ aplicarse.

- Evaluación individual a partir de desempeño.

- Evaluación grupal considerando la articulación de construcciones individuales y grupales

- Etc.

\subsection{Análisis de la experiencia}

Mirando retrospectivamente, consideramos que el enfoque y la modalidad adoptada contribuyeron al logro de los objetivos propuestos. En este marco resaltamos que

Los cambios introducidos en el contenido y las actividades:

- Aportaron el entorno propicio a la naturaleza de los «nuevos» contenidos y procedimientos de investigación.

- Ofrecieron variedad de recursos didácticos y soportes para el contenido y las actividades.

- Generaron nuevos formatos de interacción de los estudiantes con el contenido y nuevas relaciones entre el contenido y la tarea correspondiente.

- Facilitaron experiencias de acceso autónomo al contenido y trabajo crítico y constructivo del tiempo presencial.

- Contribuyeron a desarrollar prácticas propias de la investigación, diferentes de las tradicionales.

Por otro lado destacamos que la propuesta de trabajo en relación a los procesos de comunicación

- Estimuló la comunicación interpersonal.

- Planteó diversas interacciones entre docentes y estudiantes y entre los estudiantes entre si

- Posibilitó compartir e integrar perspectivas.

- Facilitó la comunicación con los alumnos en: aspectos formales, académicos y cotidianos.

y en relación a la evaluación facilitó la tarea de evaluación y seguimiento en tanto que generó registros automáticos fundamentalmente y contribuyo a la retroalimentación continua. 
Cabe también destacar de la experiencia nos dejó importantes interrogantes respecto a las condiciones y estrategias a seguir para aprovechar la oportunidad de trabajo. En esta línea es importante señalar aspectos que consideramos sensibles o críticos para los involucrados en el proceso, como los siguientes:

- Alumnos poco familiarizados con tecnología en los espacios académicos.

- Resistencia a romper a romper la inercia de la clase tradicional consolidada durante muchos años en el espacio institucional y en ocasiones desorientación para participar.

- "Temor a mostrarse».

- Tendencia a las conversaciones lineales (en los foros) con escasos intercambios alumno-alumno, evidenciando temor de interpelar a los compañeros, de mostrar otros puntos de vista, de criticar una actividad y dejar constancia de ello por escrito.

- Tendencia a priorizar las exigencias de lo presencial sobre las del aula virtual y desplazamiento de actividades autorreguladas.

La carga horaria de los estudiantes, el exceso de tareas académicas, y la libertad de escoger que y cuando lo hacían a lo largo del año dificultó el desarrollo de los trabajos en los tiempos esperados, eneró disparidad de ritmos de aprendizaje.

\subsection{A modo de conclusión de la propia experiencia presentamos extractos de un foro desarrollado en el marco de la asignatura.}

La construcción de conocimiento en la era digital supone nuevas formas de abordar epistemológicamente las prácticas investigativas, profesionales y docentes. Actualmente estamos atravesados por infinidad de medios a través de los cuales podemos acceder a la información, pero como sujetos de la educación tenemos la responsabilidad de apropiarnos de este contenido de forma crítica, constructiva y atendiendo a los sujetos que los construyen.

La Investigación Educativa juega un papel esencial a la hora de plantear problemáticas que permitan poner en juego estas nuevas formas de acceder y construir conocimiento, puede permitirnos ampliar nuestras miradas hacia nuevos horizontes de posibilidad y no sólo quedarnos con las prácticas tradicionales.

El nuevo contexto abre posibilidades que la investigación puede abonar y que la investigación misma tiene que aprender a andar. Sin procesos sistemáticos de análisis y reflexión difícilmente puede haber apropiación crítica y construcción más allá de lo habitual. (IEDUCATIVA, 2015)

\section{Conclusión General}

Desde lo trabajado, observamos la diversidad de formas de entender la formación en metodología de la investigación educativa en Latinoamérica, y dentro de esa diversidad, los elementos limitantes que mantienen las miradas tradicionales. Las TIC son incorporadas solo testimonial u ocasionalmente, parece que la sociedad y la educación pertinente a ella no hubiera cambiado en los últimos 30 años.

Formación en investigación educativa en la sociedad digital. Una experiencia innovadora de enseñanza en el nivel superior. Selín Carrasco, Silvia Baldivieso y Lorena Di Lorenzo. 
La discusión acerca del triple rol de las TIC en la Sociedad Actual, o la incorporación de lo virtual como una dimensión mas en la vida humana, no son consideradas.

Concepciones de la sociedad actual que deberían influir en las investigaciones y en la formación de investigadores tales como Sociedad Red, Sociedad Líquida, Sociedad compleja son raramente contemplados. La formación en Metodología de la Investigación mantiene una mirada epistemológica de mediados del siglo XX y de ello se desprende que la Investigación resultante no sirve como dispositivo de pilotaje para mirar hacia adelante.

Desde lo vivencial, observamos la resistencia de las comunidades de investigación educativa a los cambios y a su necesidad de aferrarse a esquemas que les permiten mantener las propias áreas de confort.

América Latina requiere ampliar su mirada en la formación de investigadores, situados en la sociedad latinoamericana actual, que tiene su propia forma de evolucionar, diferente a la europea o asiática por decir, pues es fundamental que estos investigadores no sólo puedan observar lo que ocurre en el momento, la fotografía instantánea, sino proyectar los resultados de su investigación hacia una sociedad en que lo digital es ya un elemento cultural.

Se impone avanzar hacia una epistemología y por ende un lenguaje propio de la educación y la investigación en la sociedad de la información. La investigación educativa necesita ser pionera en este cambio de paradigmas sociales, para poder enseñar a problematizar su realidad y construir realidades; necesita crear su propio lenguaje y conocimiento que le permita avanzar hacia educaciones e investigaciones pertinentes al contexto.

Se impone rediseñar procesos de formación a la luz del análisis de experiencias y continuar en la búsqueda de nuevas formas de formación en investigación articulando tecnología e investigación es espacios reales de educación.

Presentación del artículo: 17 de diciembre de 2015

Fecha de aprobación: 22 de diciembre de 2015

Fecha de publicación: 30 de enero de 2016

Carrasco, S., Baldivieso, S. y Di Lorenzo, L. (2016). Formación en investigación educativa en la sociedad digital. Una experiencia innovadora de enseñanza en el nivel superior en el contexto latinoamericano. RED. Revista de Educación a Distancia. 48(6). Consultado el (dd/mm/aaaa) en http://www.um.es/ead/red/48/selin_et_al.pdf

\section{Bibliografía}

Aaron S., et al. (2014) What Is Flipped Learning? Flipped Learning Network (FLN).

The Four Pillars of F-L-I-PTM . Recuperado de http://www.flippedlearning.org/cms/lib07/VA01923112/Centricity/Domain/46/FLIP _handout_FNL_Web.pdf 
Arizona State University. (1999) Education Policy Analysis Archives, Volume 7, Number 2 January 23.

Baldivieso, S. (2012). Bases del Modelo Pedagógico de la Escuela de Pedagogía de la Universidad de La Frontera. Temuco: Escuela de PedagogíaUniversidad de la Frontera. Recuperado de http://fliphtml5.com/agvd/ugyv/basic

Becher, T. (2001). Tribus y territorios académicos. Barcelona: Gedisa.

Boaventura de Sousa, S (2009). Un discurso sobre las ciencias. en Boaventura de Sousa Santos. Una epistemología del sur: la reinvención del conocimiento y la emancipación. México: Siglo XXI-CLACSO.

Carrasco, S. (2011, septiembre 29-octubre 1). "Los desafíos de la Educación en la Sociedad del conocimiento". En Congreso Internacional de Educación 2011. San Luis, Argentina. Recuperado de http://blogcued.blogspot.com.ar/2011/10/congresointernacional-de-educacion.html

Carrasco, S. y Baldivieso, S. (2014). "Los recursos de aprendizaje en la educación a distancia, nuevos escenarios experiencias y tendencias”, en: Rama, Claudio y Ramírez Fidel. Los recursos de Aprendizaje en la educación a distancia. Montevideo: Virtual Educa.

Castells, M. (2006). La Sociedad Red. Madrid: Alianza Editorial.

Didou-Aupetit, S. (2007), Evaluación de la productividad científica y reestructuración de los sistemas universitarios de investigación en América Latina. Revista educación superior y sociedad: nueva época, Serie 12, 1. Venezuela: IESALC.

Di Lorenzo, L. (2015). Significados que los egresados del profesorado en ciencias de la educación le atribuyen a la formación docente en pensamiento crítico. Tesis de Maestría en ciencias sociales con mención en investigación social. Córdoba, Argentina: Escuela de Trabajo Social. Universidad Nacional de Córdoba.

Echeverría J. (2001, enero 17). Sociedad y Nuevas Tecnologías en el Siglo XXI. Conferencia en Málaga. Recuperado de http://redaprenderycambiar.com.ar/javierecheverria-tercer-entorno.

García-Guadilla, C. (1998). La investigación sobre la Universidad latinoamericana desde la segunda mitad del Siglo XX. En Téllez, M., Educación, cultura y política. Ensayos para la comprensión de la historia de la educación en América Latina. Caracas: Universidad Central de Venezuela.

González, A. y otros. (2012). Propuestas educativas mediadas por tecnologías digitales: Algunas pautas de trabajo. La Plata: Universidad Nacional de La Plata. Recuperado de http://sedici.unlp.edu.ar/bitstream/handle/10915/25803/Documento_completo__pdf? sequence $=3$ 
Hernández-Jácquez, L.F. y Barraza-Macías, A. (2015). Autoeficacia académica y estrés. Análisis de su relación en estudiantes de posgrado. México: Universidad Pedagógica de Durango.

IEDUCATIVA. (2015, noviembre 12-19) : II Foro de Investigación educativa "Las preguntas de la investigación educativa hoy". [Foro virtual]. Organizado por la asignatura Investigación Educativa II, de la Facultad de Ciencias Humanas. UNSL. Recuperado de http://ieducativa.org/2015/11/ii-foro/

Messina G. (2004). La sistematización educativa acerca de su especificidad. Revista enfoques educacionale., Universidad de Chile 6 (1) 19 -28.

Morin, E. (2006). Inteligencia de la Complejidad, Epistemología y Pragmática. Luxemburgo-Francia: Ediciones de l'Aube.

Narodowski, M. (1999) “La Investigación Educativa en América Latina:Una Respuesta a Akkari y Perez"Investigación en educación en América Latina: una continuación del debate. Vol 7 Num 10 Recuperado de http://www.quadernsdigitals.net

Palamidessi, M. I., Gorostiaga, J. M. y Suasnábar, C. (2014). El desarrollo de la investigación educativa y sus vinculaciones con el gobierno de la educación en América Latina., Perfiles Educativos, vol. XXXVI, núm. 143, 2014, pp. 49-66, México D.F.: Instituto de Investigaciones sobre la Universidad y la Educación.

Piñero M. y Rivera M. (2004): Transversalidad e integración de competencias específicas en, por y para las TIC y la investigación en la formación de formadores. Multiciencias. Vol. 10 Nro. 1.

Rincón, C. (2004). La formación de investigadores en educación: retos y perspectivas para América Latina en el siglo XXI. Revista Iberoamericana de Educación. Enero.

Rojas-Soriano, R. (2015). Aspectos tóricos sobre el proceso de formación de investigadores sociales. En Abero, L., et al. Investigación educativa abriendo puertas al conocimiento. Buenos Aires: CLACSO.

Sanchez-Puente, R. (2014). Enseñar a investigar. Una didáctica nueva de la investigación México: IISUE.

Sirvent, M.T. (2006) "El Proceso de Investigación”. [Documento de Cátedra]. Buenos Aires: Facultad de Filosofía y Letras, Departamento de Ciencias de la Educación Investigación y Estadística educacional. Universidad Nacional de Buenos Aires.

Sirvent, María-Teresa, (2015, agosto 12-14). Enseñar a investigar en la universidad. Contextos, propósitos y desafíos en la formación metodológica de grado y de posgrado. [Presentación]. Seminario Internacional de Educação Superior - RIES Rede GEU, Faculdade de Educação de la Universidade Federal do Rio Grande do Sul, Porto Alegre,. 
Solis-Alpízar, M. et al. (2012, octubre). La investigación educativa en los procesos de formación inicial docente de primaria en universidades públicas y privadas de Costa Rica. Costa Rica: Ministerio de Educación Pública. Recuperado de http://www.mep.go.cr/sites/default/files/formacion_inicial_docente.pdf 